Abschließungen gegenüber der Außenwelt führen, die durch eine enge Kooperation zwischen den sich formierenden Handelsblöcken - vielleicht gerade in der Frage der Entwicklungshilfe - bekämpft werden müßten.

Bernhard Großmann

\title{
JOHN BADGLeY
}

\section{Asian Development. Problems and Prognosis}

The Free Press, New York, London 1971, XIV, 210 Seiten

An den Anfang seines Buches, das ein Buch über Asien sein soll, aber allenfalls eines über 13 südostasiatische Länder ist, stellt der Politologe Badgley die Worte eines westlichen Poeten (Goethe) und eines östlichen Politikers (Soedjatmoko), wobei letzterer u. a. sagt: "... one of the greatest problems they (the people in Southeast Asia) are facing is the apparent lack of relationship between problems presented by the external view of the development process and the way the interior view presents them." Sein Buch versteht Badgley als (partielle) Antwort hierauf, als interpretative Evaluierung des politischen Wandels („interor view“) den südostasiatischen Ländern und dessen Implikationen für den Politiker innerhalb wie außerhalb dieser Länderecke.

In einem ersten Teil werden das bisherige und zukünftige asiatische Milieu analysiert und prognostiziert, in einem zweiten Teil die bisherige und zukünftig mögliche oder notwendige Entwicklungs- und Sicherheitspolitik. Einleitend geht der Autor mit der Meinung ins Gericht, Wandel in den asiatischen Ländern durch amerikanische Aktionen herbeiführen oder lenken zu können; Vietnam sieht er als Katalysator allgemeiner (?) Erkenntnis westlicher politischer Impotenz. Zugleich taucht hier das "Leitmotiv“ des Buches auf, die Infragestellung (a) der These vom nation-state als bester Strategie für gelenkten (wirtschaftlichen, sozialen und politischen) Wandel und der Gleichsetzung von Zentralisierung mit Entwicklung und (b) der (eindimensionalen) Überbetonung des supranationalen regionalism - beide zusammen die Grundlagen bisheriger amerikanischer Asienpolitik, von der Annahme einer chinesischen Invasions- oder Subversionsgefahr bestärkt („. . . the US government still believes that, without the strength of numbers, the states of Southeast Asia will be coerced into subservience by their giant neighbors") (S. 192). Badg!ey hält es als iilustrativ für das westliche Verständnis der asiatischen Situation, daß selbst Autoren wie Gunnar Myrdal (Asian Drama), Theodore Geiger (The Conflicted Relationship) und Lucian Pye (Aspects of Political Development) die Möglichkeit übersehen, daß nation-building selbst ein entscheidendes Hindernis für Entwicklung sein kann.

Dagegen konstatiert er für die (südost)asiatischen Länder ein zunehmend divergierendes Lokalbewußtsein, einen stärker werdenden Hang zur lokal-regionalen Autonomie, eine wachsende Segregation zwischen Elite bzw. Bürokratie und der großen Masse des Volkes, speziell der Bauernschaft, eine stärker werdende Isolierug der meisten Zentralregierungen von der Basis im Falle ernstlicher Attacken von oppositioneller Seite und einen allgemeiner werdenden Mangel an Autorität im zivilen Bereich. Diese Trends sind für ihn Anzeichen dafür, daß der Prozeß des politischen Wandels in den (südost)asiatischen Ländern sich weiter verlangsamen werde. Um den langfristigen Interessen der zwei Milliarden Asiaten von heute (und der vier Milliarden in 25 Jahren) zu dienen, sei daher die Frage zu überdenken, wo Souveränität und Autorität innerhalb der staatlichen Gebilde zu lokalisieren 
bzw. wohin zu verlagern sei. Der Staat, den die meisten (südost)asiatischen Regierungen repräsentieren, sei jedenfalls der ländlichen Bevölkerung ebenso fremd wie andere westliche, von Kolonialisten und heimischen Intellektuellen eingeführten Institutionen.

Bis hierher, vor allem aber auch nach der Lektüre der Kritik am voreiligen supranationalen regionalism und an den multinationalen Organisationen im südostasiatischen Raum ist man geneigt anzunehmen, der Autor folge (zumindest indirekt) der Meinung Johan Galtungs, daß die asiatischen Länder nicht nur sehr verschieden und desintegriert sind, sondern, daß es vielmehr ein Gebilde „Asien“ gar nicht gibt: „Asien ein Mythos!“. Doch formuliert der Autor in mehreren Kapiteln seine Taxonomie der Krise und des Konflikts von mehr bzw. weniger lebensfähigen asiatischen Staaten auf der Basis von Trendextrapolationen üblicherweise verwendeter Durchschnittsgrößen und ihrer Wachstumsraten (wie Bruttosozialprodukt, Industrieproduktion, Energieverbrauch usw.), deren theoretische und empirische Aussagefähigkeit, weil auf die Nation bezogen, er vorher selbst implizite verneint hat. Durch Sondierung von Machtelementen und ihrer Gewichttung und durch Bewertung von Entwicklungshemmnissen typisiert Badgley dann die "Lebensfähigkeit" (viability) von 13 asiatischen Ländern wie folgt: sie sei „hoch" für: Südkorea, Taiwan, Thailand; „bescheiden“ für: Malaysia, Nordkorea, Nordvietnam, Philippinen; „niedrig“ für: Burma, Kambodscha, Indonesien, Laos; "nicht vorausschaubar" für: Singapur, Südvietnam.

In den folgenden Kapiteln werden zukünftig mögliche Konfliktsituationen in den asiatischen Ländern behandelt und eine Erweiterung des Leitmotivs vorgenommen, die konzeptionellen Probleme der Entwicklung, nation-building und regionalism. Interessant ist der Versuch der Identifizierung und Typisierung von Konflikten und deren Ursachen, während die Frage alternativer Konzepte letztlich an der Unbestimmtheit des Entwicklungsbegriffs scheitert. Fixiert auf das Problem von Bevölkerungsexplosion und zurückbleibender Verwaltungs- und Regierungsfähigkeit ("a central government attempting to command the pace of change... of ten, thirty or some hundred million people is bound to be overextended and unresponsive”), enden seine Alternativvorschläge in wenig greifbaren Begriffen, wie: „indigenous pluralism“, „organization of diversity“, „communality“, „civil polity“ oder "modernity of tradition“.

Damit bleibt aber insbesondere offen, ob Zentralisierung versus Dezentralisierung a priori entschieden werden kann oder ob es nicht von Form, Reichweite und Identifizierung mit konkreten Zielen abhängt, das „konzeptionelle Problem“ also primär ein Ziel- und weniger ein Methodenproblem ist. Dies hätte für den Autor in diesem Falle auch bedeuten müssen, sich intensiver mit Japan und China auseinanderzusetzen; doch hier unterliegt er mehr als einmal dem intellektuellen Problem des westlichen außenstehenden Asienwissenschaftlers, der eigenen Voreingenommenheit oder schlicht falscher Information: "China's development prospects are especially dim, suggesting continued dissatisfaction with any central government, and the possibility of the more productive provinces developing autonomous political systems to protect themselves against the drain of the poorer regions" (S. 6). Selbst wenn der erste Halbsatz stimmen würde, wäre der zweite Halbsatz schließlich der Rat zur Dezentralisierung, den Badgley selbst den südostasiatischen Ländern in der Konsequenz bietet. Zumindest die OOkonomen unter den Chinawissenschaftlern scheinen sich indes in einer Sache einig zu sein, daß es bisher nur wenige vergleichbare Versuche, zu einer ausgeglicheneren internen regionalen Ent- 
wicklung zu kommen, gegeben hat; die ehemalige Kopflastigkeit der regionalen Wirtschaftsstruktur des Landes ist ohne $Z$ weifel nicht mehr vorhanden.

Udo E. Simonis

Vinoba Bhave

Struktur und Technik des inneren Friedens

Bellnhausen/Hessen 1971, 174 Seiten, DM 12,80

Verlag Hinder und Deelmann

Im Jahre 1944 wurde Vinoba Bhave wegen Teilnahme an der Nationalbewegung Ghandis von den Kolonialbehörden verhaftet und ins Gefängnis von Seoni eingeliefert. Dort durfte er seinen Mitgefangenen Vorträge über die geistigen Grundlagen indischer Weltanschauung halten, äußerlich an Verse der Bhagavad-Gita anknüpfend. Die Vorträge auf Marathi wurden später zusammengefaßt und in viele indische Sprachen übersetzt, 1965 auch ins Englische (The steadfast Wisdom). Dieser englischen Ausgabe liegt die vorliegende deutsche Übersetzung zugrunde. Der ursprüngliche Titel „Sthita-prajñādaršana“ (Anschauung einer dauerhaften Weisheit) besagt im indischen Sprachgebrauch, daß hier ein anschaulisches System entwickelt wird, welches den Menschen unverlierbar befähigt, sein Leben durch Selbsterziehung weise zu meistern. Weisheit (prajñā) ist im Indischen eine Haltung inneren Friedens, durch welche die Probleme des Lebens richtig überblickt werden können; und Anschauung (daršana) ist der Name für jedes philosophische System, das Theorie und Praxis umfaßt. Von daher ist der deutsche Titel eine erläuternde Ubersetzung des in seiner Kürze kaum erfaßbaren indischen Titels.

Der Grundgedanke des Buches ist, daß der äußere politische Friede, für den Vinoba Bhave als Mitarbeiter Ghandis damals kämpfte und für den er als Initiator der Landschenkungsbewegung noch heute wirksam eintritt, nur durch den inneren Frieden des einzelnen garantiert wird. So schließt der deutsche Herausgeber sein Vorwort wie folgt ab:

„Ohne daß der einzelne den nach innen verlagerten und den in seinem Innern ohnehin tobenden Kampf ausgekämpft hat, ist seine Friedfertigkeit nicht verläßlich. Von daher hat die Gewinnung des inneren Friedens sowie die Kenntnis von dessen Wesen eine unschätzbare Bedeutung für die Sicherung des äußeren Friedens und mithin für die Friedensforschung und Politik" (S. 9).

Das Buch gliedert sich in $20 \mathrm{Kapitel}$, die in insgesamt 200 Punkte aufgegliedert sind. Es dürfte für den an europäische Denkschemata Gewohnten nicht gerade einfach sein, den Zugang zum praktischen Nutzen der philosophischen Ausführungen und Begründungen des Autors zu finden. $\mathrm{Da}$ sie einen solchen Nutzen für den Inder hatten und haben, steht jedoch außer Zweifel. Das Buch mag daher anregen, die existentiellen Fragestellungen, die hier entrollt werden, nachzuvollziehen, um so die abendländische Anschauungsweise zu erweitern. Eine gewisse Verbindung zwischen den Grundgedanken von Platons "Gastmahl“ oder seinem "Staat" sowie der Haltung der Stoa einerseits und Vinoba Bhaves Einstellung andererseits drängt sich auf. Er regt an, „daß die verantwortliche Führung Menschen immerwährender Weisheit anvertraut" und daß „die soziale Ethik auf Selbstkontrolle gegründet" wird: „So wird eine gesellschaftliche Ordnung entstehen, die zum sozialen Gleichgewicht führt. Nur so können Zufriedenheit und Frieden erreicht werden" (S. 98). „Friede stellt sich von selbst ein, wenn unser gesellschaftliches Bewußtsein in der steten Weisheit gründet“ (S. 98). Immer wieder wird sein Grundgedanke von ver- 